\title{
Effect of Different Post-Harvest Treatments on the Storage Quality of Peach cv. July Elberta
}

\author{
L. Sophia Devi ${ }^{1 *}$ and S.P.S. Gulleria ${ }^{2}$ \\ ${ }^{1}$ Directorate of Research, CAU-Imphal, Manipur, India \\ ${ }^{2}$ Department of Post-Harvest Technology, Dr Y S Parmar University of Horticulture and \\ Forestry, Nauni-Solan-173230, Himachal Pradesh, India \\ *Corresponding author
}

\section{A B S T R A C T}

The study was undertaken to assess the effect of various post-harvest treatments on the storage quality of peach cv. July Elberta. Fruits for this experiment were procured from

\section{Keywords}

Postharvest coating, Storage quality, Neem leaf extract, Rice starch, Cassava starch

Article Info

Accepted: 02 May 2018 Available Online: 10 June 2018 trees which were maintained under a uniform cultural schedule throughout the season. Fruits were harvested at proper maturity and packed in CFB cartons for their transportation to the laboratory for conducting the studies. For the application of postharvest coating treatments, unblemished, uniform and large sized fruits were selected and washed under a clean tap water. After air drying, these fruits were treated with different post-harvest coating materials like rice starch, cassava starch, neem leaf extract at three different concentrations by dipping them in the solution for 5 minutes. During the present investigation, the extent of physiological weight loss was, however, found to be lower in precooled fruits as compared to those that were not precooled. The application of coating materials further decreased physiological weight loss; whereas; it was maximum in control fruits. Among post-harvest edible coating treatments so adopted, 30 per cent neem leaf extract was most effective in minimizing physiological weight loss, firmness, acidity, pectin; and thereby, exhibiting maximum overall acceptability.

\section{Introduction}

Peach (Prunus persica L. Batch) is an important stone fruit grown under temperate and sub-tropical climate. In India, peach cultivation extends from Northern plains to an elevation of 2000 metres above mean sea level occupying an area of about 18,500 ha with an annual production of 1,50,000 MT (Soodan et al., 1994, NHB, 2014). It is commercially grown in states of Arunachal Pradesh, Haryana, Himachal Pradesh, Jammu and Kashmir, Nagaland, Punjab, Uttarakhand and Uttar Pradesh. However, in Himachal Pradesh peach is cultivated commercially in an approximate area of 4800 ha, which is scattered all over the state except the dry and cold regions of Lahaul and Spiti, Kinnaur, Pangi and Bharmour areas of Chamba district, with an annual production of about 6564 MT (Anon 2014). July Elberta is the largest propagated commercial cultivar grown in Himachal Pradesh due to its high yield and good market value. 
Peaches like other stone fruits cannot endure long storage life at normal atmosphere and ambient temperature after harvest as the fruit tends to soften, thereby, resulting in textural breakdown in postharvest period of handling and transportation. The losses in stone fruits may be higher as these are highly deteriorative in nature.

Peaches exhibit considerable deterioration in quality after harvest due to rapid physiological changes which continue to occur in the fruit during marketing and storage. Further, the harvest season coincides with peak summers or early monsoon, which favours the growth and multiplication of spoilage causing organisms which ultimately results in lowering the fruit quality and life as well as consumer acceptability thereby tending to cause a huge economical loss to the growers. These physiological changes influence the appearance, colour, texture, flavour, etc. of the fruit, thereby affecting their overall quality and marketability. Most of these changes are undesirable from the point of view sale promotion and have to be slowed down to get quality fruit after storage and handling.

Strategically, the removal of field heat by precooling is the fundamental process in the marketing of fruits as this increases the shelflife of the produce, thereby allowing the growers to send their produce to distant markets to fetch better prices. Also, the coating of fruits with different substances also holds promise as it can be done immediately after harvesting the fruits as the skin coatings form a thin semi permeable membrane to regulate the diffusion of $\mathrm{O}_{2}$ and $\mathrm{CO}_{2}$ into and out of the fruit and which also provide natural barrier to the commodity. Among these strategies, apart from waxing the use of edible material for this purpose is a relatively a new approach which is emerging as an effective treatment in reducing post-harvest spoilage though scattered attempts have been made. Yet information on these attempts for regulating quality and life of stone fruits is totally lacking and hence the felt need to exploit such efforts in peaches. Thus there is the need to investigate further, the effect of different postharvest treatments combinations with respect to concentrations on quality and shelf life of peach cv. July Elberta.

\section{Materials and Methods}

Fruits for this experiment were procured from trees which were maintained under a uniform cultural schedule throughout the season. Fruits were harvested at proper maturity and packed in CFB cartons for their transportation to the laboratory for conducting the studies. On arrival in the laboratory, the fruits were inspected and injured/blemished fruits were culled out. The fruits were then divided into two lots.

One of the lots was subjected to precooling in a low pressure container. The other lot of fruits was not precooled. Fruits from both the lots were divided into 30 parts for the application of 3 different coating treatments in three replications as per the details, without precooling treatments $\left(\mathrm{T}_{1}\right.$ : Rice starch $1 \%+$ Guar gum 2\%, $\mathrm{T}_{2}$ : Rice starch $2 \%+$ Guar gum $2 \%, \mathrm{~T}_{3}$ : Rice starch $3 \%+$ Guar gum $2 \%$, $\mathrm{T}_{4}$ : Cassava starch $1 \%+$ Guar gum $2 \%, \mathrm{~T}_{5}$ : Cassava starch 2\% + Guar gum 2\%, $\mathrm{T}_{6}$ : Cassava starch 3\% + Guar gum 2\%, $\mathrm{T}_{7}$ : Neem leaf extract $10 \%, \mathrm{~T}_{8}$ : Neem leaf extract $20 \%$, $\mathrm{T}_{9}$ : Neem leaf extract $30 \%$ and $\mathrm{T}_{10}$ with water dip only) and with precooling treatments $\left(\mathrm{T}_{1}\right.$ : Rice starch 1\% +Guar gum 2\%, $\mathrm{T}_{2}$ : Rice starch $2 \%+$ Guar gum $2 \%, \mathrm{~T}_{3}$ : Rice starch $3 \%$ + Guar gum 2\%, $\mathrm{T}_{4}$ : Cassava starch $1 \%+$ Guar gum 2\%, $\mathrm{T}_{5}$ : Cassava starch $2 \%+$ Guar gum 2\%, $\mathrm{T}_{6}$ : Cassava starch $3 \%+$ Guar gum $2 \%, \mathrm{~T}_{7}$ : Neem leaf extract 10\%, $\mathrm{T}_{8}$ : Neem leaf extract $20 \%, \mathrm{~T}_{9}$ : Neem leaf extract $30 \%$ and $\mathrm{T}_{10}$ with water dip only). 
For the application of postharvest coating treatments, unblemished, uniform and large sized fruits were selected and washed under a clean tap water. After air drying, these fruits were treated with different coating materials by dipping them in the solution for 5 minutes. The control fruits under treatment $\left(\mathrm{T}_{10}\right)$ were simply dipped in distilled water. The coated fruits were placed on newspaper sheets under a fan for air drying in a shade at room temperature. The sample fruits were packed in corrugated fibre board cartons with paper trays and stored under refrigerated conditions maintained at $3+1^{\circ} \mathrm{C}$ and $80-85$ per cent relative humidity, during the entire storage period and was conducted in the postharvest and physiology laboratory of the Department of Postharvest Technology, College of Horticulture, Dr Y. S. Parmar University of Horticulture and Forestry Nauni, Solan (H.P.) and stored. Observations regarding physicochemical characteristics viz. physiological loss in weight (PLW), firmness, total soluble solids (TSS), titratable acidity, ascorbic acid, total sugar, reducing sugar and pectin content of fruit were recorded at an interval of one week during the storage period. Acidity was measured in terms of $\%$ malic acid. Ascorbic acid, total and reducing sugar were estimated by standard methods (AOAC 2000). Pectin content in fruit was determined according to the standard procedure (Ranganna 1986). Fruit firmness was measured with an Effigy Penetrometer (model FT 327), which recorded the pressure required to force a plunger of $11 \mathrm{~mm}$ diameter into tared flesh of fruit samples. The readings were taken on diagonally opposite sides of each fruit and results expressed in Newton $(\mathrm{N})$.

Newton $=$ lbs/sq. inch $\mathrm{x} 4.448$

Observations regarding physico-chemical characteristics of fruit were recorded at an interval of one week during the storage period. Data on physico-chemical characteristics of fruits were analysed statistically by Completely Randomized Design (CRD) during storage.

\section{Results and Discussion}

\section{Physiological Loss in Weight (PLW)}

Physiological loss in weight (PLW) increased with increasing period of storage in all the treatments for both precooled and nonprecooled fruits as indicated in table 1. Among treatments, minimum loss in physiological weight loss was observed in precooled fruits treated with 30 per cent Neem leaf extract $\left(\mathrm{T}_{9}\right)$. Coating of plant leaf extracts might form a thin film around each fruit, which can act as a semi permeable membranes to regulate the diffusion of $\mathrm{O}_{2}$ and $\mathrm{CO}_{2}$ into and out of the fruits; and, thereby, reducing the rate of metabolism and also prevents water loss (Smith and Stow, 1984 and Bhardwaj and Sen, 2003). The application of coating materials further decreased physiological weight loss; whereas it was maximum in control fruits $(21.22 \%)$ that were not subjected to precooling. The lowest mean physiological weight loss (7.99\%) was recorded in response to 30 per cent Neem leaf extract coating with precooling; and that was followed by 20 per cent Neem leaf extract ( $\mathrm{T}_{8}$ with $8.30 \%$ ) with precooling treatment. Also, Lowing and Cutts (1982) have provided supportive evidence to the present findings when they opined that coatings provide a partial barrier to moisture and gas exchange and produce a modified atmosphere within the coated fruits; and, thereby, reducing respiration and transpiration. At the same time, the scientific explanation advanced by Saftner (1999) also holds good in the present investigation; and, whereas; it has been rightly concluded that fruit coatings tend to have high permeability for $\mathrm{CO}_{2}$ and $\mathrm{O}_{2}$ and relatively low permeability for water vapour. 


\section{Fruit firmness}

Fruit firmness, as affected by precooling and postharvest coating treatments during cold storage revealed a gradual and significant decrease in fruit firmness with the advancement of storage duration (Table 2). However, precooled fruits with 30 per cent Neem leaf extract $\left(T_{9}\right)$ proved to be the most effective treatment in retaining fruit firmness $(49.90 \mathrm{~N})$ when combined with precooling; and that was followed by $\mathrm{T}_{3}(49.85 \mathrm{~N}), \mathrm{T}_{8}$ $(49.78 \mathrm{~N})$ and $\mathrm{T}_{2}(49.48 \mathrm{~N})$, respectively.

Retention of relatively high firmness under this treatment could be due to slower metabolic activities leading to slower ripening changes and delayed senescence. The present findings are also in confirmation with the studies of Sornisrivichai et al., (1990) who reported that waxing/coating treatment to pear fruits slow down the decrease in firmness, and Combrink and Visagie (1997) have also lend support to our results that precooling improved the fruit structure with regard to fruit firmness as these researchers have natively found that partial precooling of apricots, peaches, nectarines and plums helped in maintaining the firmness of fruit and the fruits that were not precooled softened quickly. The loss of pectin substances from the middle lamella of the cell wall is perhaps the key step in the ripening process that leads to the loss of cell wall integrity of fruits (Gross and Sams, 1984) and consequently leads to softening. All these explanation lend conclusive evidence to our finding that the precooling coupled with surface coatings provided a relative decreased in firmness during the storage intervals.

\section{Total Soluble Solids (TSS)}

The data on total soluble solids (TSS) content of peach fruits as affected by various coating and precooling treatments revealed that the
TSS content increased with the increased storage period and then declined towards the final stage of storage (Table 3). The increase in TSS and sugar contents during the earlier part of storage may be due to the hydrolysis of insoluble polysaccharides into simple sugar.

Such changes are expected to be slower and more gradual when the metabolism, of the commodity is slowed down by the application of various treatments.

The increase in TSS and sugar contents in control fruits may be due to the higher physiological weight loss in these fruits, as a result of which there might have been an increase in the concentration of sugars. An increase in TSS was also reported by Vanoli et al., (1995) in peach during storage. The fruits which were not precooled had an overall mean TSS content (11.04\%) as compared to TSS of fruits (11.14\%) which were subjected to precooling.

Among the treatments, highest mean TSS content $(12.14 \%)$ was recorded in control fruits, which was followed by 1 per cent Cassava starch $\left(\mathrm{T}_{4}\right)$ with $11.22 \%, 1 \%$ Rice starch $\left(\mathrm{T}_{1}\right)$ with $11.09 \%, 2 \%$ Cassava starch $\left(\mathrm{T}_{5}\right)$ with $11.05 \%$ and $2 \%$ Rice starch $\left(\mathrm{T}_{2}\right)$ with $11.03 \%$, total soluble solids, respectively.

Precooling and edible coating treatments might have also slowed down the conversion of starch into simple sugars and also the respiration rate of fruits as a result of which a decrease in these constituents was not observed during the last week of storage in both the storage conditions.

Similar slowing down of changes in TSS and sugar contents as a results of coating treatments with rice starch and Neem leaf extract have also been reported by various 
workers (Singh and Mohammed, 1997 and reasonings make the results so obtained Singh et al., 2000) and these agreeable acceptable and meaningful.

Table.1 Effect of various post-harvest treatments on the physiological weight loss (\%) of peach cv. July Elberta fruit during storage at $3 \pm 1^{\circ} \mathrm{C}$

\begin{tabular}{|c|c|c|c|c|c|c|}
\hline \multirow[t]{2}{*}{ Treatments $(\mathrm{T})$} & \multicolumn{6}{|c|}{ Storage interval in days (I) } \\
\hline & 7 & 14 & 21 & 28 & 35 & Mean \\
\hline \multicolumn{7}{|l|}{ Without Pre-cooling $\left(\mathrm{P}_{1}\right)$} \\
\hline $\mathrm{T}_{1}$ Rice starch (1\%) & 3.81 & 6.89 & 10.21 & 13.36 & 23.62 & 11.58 \\
\hline $\mathrm{T}_{2}$ Rice starch $(2 \%)$ & 3.73 & 6.42 & 9.99 & 12.81 & 22.74 & 11.14 \\
\hline $\mathrm{T}_{3}$ Rice starch $(3 \%)$ & 3.27 & 5.56 & 9.67 & 12.41 & 22.45 & 10.67 \\
\hline $\mathrm{T}_{4}$ Cassava starch (1\%) & 3.88 & 6.64 & 10.21 & 12.97 & 23.00 & 11.34 \\
\hline $\mathrm{T}_{5}$ Cassava starch $(2 \%)$ & 3.82 & 6.53 & 10.00 & 12.90 & 22.81 & 11.21 \\
\hline $\mathrm{T}_{6}$ Cassava starch (3\%) & 3.36 & 5.69 & 9.86 & 12.52 & 22.69 & 10.82 \\
\hline $\mathrm{T}_{7}$ Neem leaf extract $(10 \%)$ & 3.30 & 5.91 & 9.85 & 12.11 & 22.50 & 10.73 \\
\hline $\mathrm{T}_{8}$ Neem leaf extract (20\%) & 3.28 & 5.71 & 9.76 & 12.06 & 22.14 & 10.59 \\
\hline $\mathrm{T}_{9}$ Neem leaf extract $(30 \%)$ & 3.23 & 5.43 & 9.52 & 12.01 & 21.75 & 10.39 \\
\hline $\mathrm{T}_{10}$ Control & 7.11 & 11.75 & 19.08 & 28.76 & 39.38 & 21.22 \\
\hline Mean & 3.88 & 6.65 & 10.81 & 14.19 & 24.31 & 11.97 \\
\hline \multicolumn{7}{|l|}{ With Pre-cooling $\left(\mathrm{P}_{2}\right)$} \\
\hline $\mathrm{T}_{1}$ Rice starch (1\%) & 2.98 & 5.54 & 8.76 & 11.09 & 17.57 & 9.19 \\
\hline $\mathrm{T}_{2}$ Rice starch $(2 \%)$ & 2.37 & 4.82 & 8.63 & 10.91 & 16.94 & 8.74 \\
\hline $\mathrm{T}_{3}$ Rice starch $(3 \%)$ & 2.16 & 4.23 & 8.43 & 10.72 & 16.07 & 8.32 \\
\hline $\mathrm{T}_{4}$ Cassava starch $(1 \%)$ & 2.41 & 4.88 & 8.66 & 11.06 & 17.00 & 8.80 \\
\hline $\mathrm{T}_{5}$ Cassava starch $(2 \%)$ & 2.29 & 4.48 & 8.64 & 10.96 & 16.56 & 8.58 \\
\hline $\mathrm{T}_{6}$ Cassava starch (3\%) & 2.20 & 4.28 & 8.52 & 10.81 & 16.16 & 8.39 \\
\hline $\mathrm{T}_{7}$ Neem leaf extract $(10 \%)$ & 2.25 & 4.50 & 8.51 & 11.00 & 16.22 & 8.50 \\
\hline $\mathrm{T}_{8}$ Neem leaf extract (20\%) & 2.13 & 4.31 & 8.23 & 10.83 & 16.00 & 8.30 \\
\hline $\mathrm{T}_{9}$ Neem leaf extract $(30 \%)$ & 2.05 & 4.23 & 8.13 & 10.07 & 15.46 & 7.99 \\
\hline $\mathrm{T}_{10}$ Control & 5.29 & 10.30 & 15.31 & 18.86 & 24.14 & 14.78 \\
\hline Mean & 2.61 & 5.15 & 9.18 & 11.63 & 17.21 & 9.16 \\
\hline \multicolumn{7}{|l|}{$\mathrm{CD}_{0.05}$} \\
\hline $\mathrm{P}$ & & & & & & 0.01 \\
\hline TxP & & & & & & 0.02 \\
\hline PxI & & & & & & 0.01 \\
\hline TxPxI & & & & & & 0.04 \\
\hline
\end{tabular}


Table.2 Effect of various post-harvest treatments on the firmness (N) of peach fruit cv. July Elberta fruit during storage at $3 \pm 1^{\circ} \mathrm{C}$

\begin{tabular}{|c|c|c|c|c|c|c|}
\hline \multirow{2}{*}{ Treatments $(\mathrm{T})$} & \multicolumn{6}{|c|}{ Storage interval in days (I) } \\
\hline & 7 & 14 & 21 & 28 & 35 & Mean \\
\hline \multicolumn{7}{|l|}{ Without Pre-cooling $\left(\mathrm{P}_{1}\right)$} \\
\hline $\mathrm{T}_{1}$ Rice starch $(1 \%)$ & 64.96 & 55.01 & 44.98 & 36.36 & 25.63 & 45.39 \\
\hline $\mathrm{T}_{2}$ Rice starch $(2 \%)$ & 65.54 & 55.22 & 45.17 & 36.53 & 26.02 & 45.70 \\
\hline $\mathrm{T}_{3}$ Rice starch $(3 \%)$ & 65.75 & 55.44 & 45.39 & 36.78 & 26.25 & 45.92 \\
\hline $\mathrm{T}_{4}$ Cassava starch $(1 \%)$ & 64.78 & 54.60 & 44.55 & 35.91 & 25.36 & 45.04 \\
\hline $\mathrm{T}_{5}$ Cassava starch $(2 \%)$ & 64.96 & 54.67 & 44.61 & 36.00 & 25.49 & 45.15 \\
\hline $\mathrm{T}_{6}$ Cassava starch $(3 \%)$ & 65.22 & 54.91 & 44.82 & 36.21 & 25.53 & 45.34 \\
\hline $\mathrm{T}_{7}$ Neem leaf extract $(10 \%)$ & 65.62 & 55.71 & 45.81 & 35.91 & 25.82 & 45.78 \\
\hline $\mathrm{T}_{8}$ Neem leaf extract (20\%) & 65.74 & 55.75 & 45.91 & 36.00 & 25.91 & 45.86 \\
\hline $\mathrm{T}_{9}$ Neem leaf extract $(30 \%)$ & 66.05 & 55.91 & 45.93 & 37.28 & 26.71 & 46.38 \\
\hline $\mathrm{T}_{10}$ Control & 54.91 & 51.30 & 40.31 & 31.33 & 23.12 & 40.20 \\
\hline Mean & 64.35 & 54.86 & 44.75 & 35.83 & 25.59 & 45.08 \\
\hline \multicolumn{7}{|l|}{ With Pre-cooling $\left(\mathrm{P}_{2}\right)$} \\
\hline $\mathrm{T}_{1}$ Rice starch (1\%) & 66.84 & 59.96 & 49.31 & 38.53 & 30.51 & 49.03 \\
\hline $\mathrm{T}_{2}$ Rice starch $(2 \%)$ & 67.34 & 60.38 & 49.73 & 38.96 & 30.96 & 49.48 \\
\hline $\mathrm{T}_{3}$ Rice starch $(3 \%)$ & 67.74 & 60.75 & 50.11 & 39.32 & 31.30 & 49.85 \\
\hline $\mathrm{T}_{4}$ Cassava starch (1\%) & 66.79 & 59.77 & 49.17 & 38.35 & 30.31 & 48.88 \\
\hline $\mathrm{T}_{5}$ Cassava starch $(2 \%)$ & 66.98 & 59.94 & 49.27 & 38.52 & 30.51 & 49.05 \\
\hline $\mathrm{T}_{6}$ Cassava starch (3\%) & 67.19 & 59.98 & 49.33 & 38.55 & 30.54 & 49.12 \\
\hline $\mathrm{T}_{7}$ Neem leaf extract $(10 \%)$ & 67.52 & 60.82 & 49.65 & 38.65 & 30.51 & 49.40 \\
\hline $\mathrm{T}_{8}$ Neem leaf extract (20\%) & 67.91 & 60.72 & 49.69 & 39.49 & 31.11 & 49.78 \\
\hline $\mathrm{T}_{9}$ Neem leaf extract $(30 \%)$ & 68.63 & 60.76 & 50.52 & 39.51 & 31.73 & 49.90 \\
\hline $\mathrm{T}_{10}$ Control & 64.83 & 54.50 & 44.26 & 32.89 & 25.74 & 44.45 \\
\hline Mean & 67.18 & 59.76 & 49.11 & 38.28 & 30.16 & 48.93 \\
\hline
\end{tabular}

$\mathrm{CD}_{0.05}$

$\begin{array}{lc}\text { P } & 0.07 \\ \text { TxP } & 0.22 \\ \text { PxI } & 0.14 \\ \text { TxPxI } & 0.47\end{array}$


Table.3 Effect of various post-harvest treatments on the TSS content (\%) of peach fruit cv. July Elberta fruit during storage at $3 \pm 1^{\circ} \mathrm{C}$

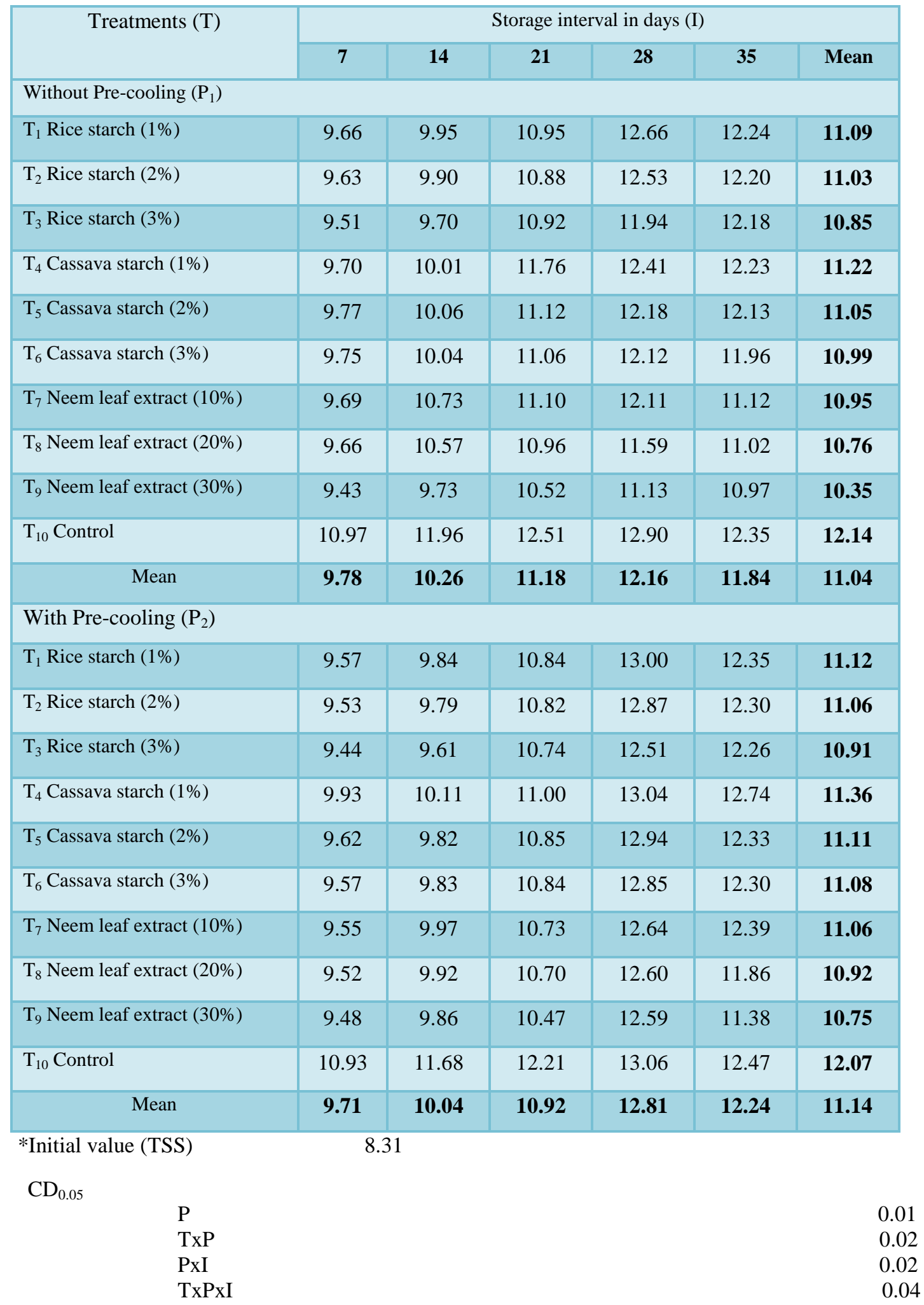


Table.4 Effect of various post-harvest treatments on the titratable acidity (\%) of peach fruit cv. July Elberta fruit during storage at $3 \pm 1^{\circ} \mathrm{C}$

\begin{tabular}{|c|c|c|c|c|c|c|}
\hline \multirow[t]{2}{*}{ Treatments $(\mathrm{T})$} & \multicolumn{6}{|c|}{ Storage interval in days (I) } \\
\hline & 7 & 14 & 21 & 28 & 35 & Mean \\
\hline \multicolumn{7}{|l|}{ Without Pre-cooling $\left(\mathrm{P}_{1}\right)$} \\
\hline $\mathrm{T}_{1}$ Rice starch (1\%) & 0.68 & 0.64 & 0.62 & 0.51 & 0.42 & 0.57 \\
\hline $\mathrm{T}_{2}$ Rice starch $(2 \%)$ & 0.67 & 0.64 & 0.63 & 0.53 & 0.49 & 0.59 \\
\hline $\mathrm{T}_{3}$ Rice starch $(3 \%)$ & 0.67 & 0.64 & 0.63 & 0.58 & 0.50 & 0.60 \\
\hline $\mathrm{T}_{4}$ Cassava starch $(1 \%)$ & 0.63 & 0.61 & 0.59 & 0.49 & 0.44 & 0.55 \\
\hline $\mathrm{T}_{5}$ Cassava starch $(2 \%)$ & 0.64 & 0.61 & 0.60 & 0.55 & 0.44 & 0.57 \\
\hline $\mathrm{T}_{6}$ Cassava starch $(3 \%)$ & 0.65 & 0.63 & 0.60 & 0.57 & 0.48 & 0.58 \\
\hline $\mathrm{T}_{7}$ Neem leaf extract $(10 \%)$ & 0.65 & 0.63 & 0.62 & 0.58 & 0.45 & 0.58 \\
\hline $\mathrm{T}_{8}$ Neem leaf extract $(20 \%)$ & 0.66 & 0.64 & 0.63 & 0.59 & 0.47 & 0.60 \\
\hline $\mathrm{T}_{9}$ Neem leaf extract $(30 \%)$ & 0.67 & 0.65 & 0.63 & 0.55 & 0.51 & 0.60 \\
\hline $\mathrm{T}_{10}$ Control & 0.58 & 0.56 & 0.54 & 0.48 & 0.42 & 0.52 \\
\hline Mean & 0.65 & 0.62 & 0.61 & 0.54 & 0.46 & 0.58 \\
\hline \multicolumn{7}{|l|}{ With Pre-cooling $\left(\mathrm{P}_{2}\right)$} \\
\hline $\mathrm{T}_{1}$ Rice starch (1\%) & 0.66 & 0.63 & 0.61 & 0.59 & 0.46 & 0.59 \\
\hline $\mathrm{T}_{2}$ Rice starch $(2 \%)$ & 0.67 & 0.64 & 0.62 & 0.58 & 0.47 & 0.60 \\
\hline $\mathrm{T}_{3}$ Rice starch $(3 \%)$ & 0.69 & 0.65 & 0.64 & 0.57 & 0.47 & 0.60 \\
\hline $\mathrm{T}_{4}$ Cassava starch $(1 \%)$ & 0.65 & 0.63 & 0.59 & 0.51 & 0.44 & 0.56 \\
\hline $\mathrm{T}_{5}$ Cassava starch $(2 \%)$ & 0.66 & 0.65 & 0.61 & 0.58 & 0.47 & 0.59 \\
\hline $\mathrm{T}_{6}$ Cassava starch $(3 \%)$ & 0.67 & 0.65 & 0.62 & 0.59 & 0.50 & 0.60 \\
\hline $\mathrm{T}_{7}$ Neem leaf extract $(10 \%)$ & 0.65 & 0.63 & 0.61 & 0.59 & 0.49 & 0.59 \\
\hline $\mathrm{T}_{8}$ Neem leaf extract $(20 \%)$ & 0.68 & 0.66 & 0.63 & 0.57 & 0.52 & 0.61 \\
\hline $\mathrm{T}_{9}$ Neem leaf extract $(30 \%)$ & 0.67 & 0.63 & 0.62 & 0.58 & 0.50 & 0.60 \\
\hline $\mathrm{T}_{10}$ Control & 0.65 & 0.63 & 0.61 & 0.58 & 0.48 & 0.59 \\
\hline Mean & 0.66 & 0.64 & 0.61 & 0.57 & 0.48 & 0.59 \\
\hline
\end{tabular}

*Initial value (Titratable acidity)

0.72

$\mathrm{CD}_{0.05}$

$\begin{array}{lc}\text { P } & 0.01 \\ \text { TxP } & 0.02 \\ \text { PxI } & 0.02 \\ \text { TxPxI } & 0.04\end{array}$


Table.5 Effect of various post-harvest treatments on the ascorbic acid content $(\mathrm{mg} / 100 \mathrm{~g})$ of peach fruit cv. July Elberta fruit during storage at $3 \pm 1^{\circ} \mathrm{C}$

\begin{tabular}{|c|c|c|c|c|c|c|}
\hline \multirow[t]{2}{*}{ Treatments $(\mathrm{T})$} & \multicolumn{6}{|c|}{ Storage interval in days } \\
\hline & 7 & 14 & 21 & 28 & 35 & Mean \\
\hline \multicolumn{7}{|l|}{ Without Pre-cooling $\left(\mathrm{P}_{1}\right)$} \\
\hline $\mathrm{T}_{1}$ Rice starch (1\%) & 3.69 & 3.50 & 3.34 & 3.30 & 3.03 & 3.37 \\
\hline $\mathrm{T}_{2}$ Rice starch $(2 \%)$ & 3.71 & 3.55 & 3.39 & 3.36 & 3.05 & 3.41 \\
\hline $\mathrm{T}_{3}$ Rice starch $(3 \%)$ & 3.73 & 3.58 & 3.44 & 3.41 & 3.07 & 3.44 \\
\hline $\mathrm{T}_{4}$ Cassava starch $(1 \%)$ & 3.60 & 3.47 & 3.37 & 3.30 & 3.04 & 3.36 \\
\hline $\mathrm{T}_{5}$ Cassava starch $(2 \%)$ & 3.65 & 3.56 & 3.40 & 3.35 & 3.07 & 3.40 \\
\hline $\mathrm{T}_{6}$ Cassava starch $(3 \%)$ & 3.74 & 3.55 & 3.43 & 3.35 & 3.11 & 3.44 \\
\hline $\mathrm{T}_{7}$ Neem leaf extract $(10 \%)$ & 3.75 & 3.51 & 3.45 & 3.40 & 3.14 & 3.45 \\
\hline $\mathrm{T}_{8}$ Neem leaf extract $(20 \%)$ & 3.77 & 3.54 & 3.47 & 3.42 & 3.16 & 3.47 \\
\hline $\mathrm{T}_{9}$ Neem leaf extract $(30 \%)$ & 3.81 & 3.60 & 3.49 & 3.44 & 3.19 & 3.50 \\
\hline $\mathrm{T}_{10}$ Control & 3.26 & 3.03 & 2.90 & 2.80 & 2.19 & 2.83 \\
\hline Mean & 3.67 & 3.49 & 3.37 & 3.31 & 3.00 & 3.37 \\
\hline \multicolumn{7}{|l|}{ With Pre-cooling $\left(\mathrm{P}_{2}\right)$} \\
\hline $\mathrm{T}_{1}$ Rice starch $(1 \%)$ & 3.87 & 3.74 & 3.62 & 3.57 & 3.37 & 3.63 \\
\hline $\mathrm{T}_{2}$ Rice starch $(2 \%)$ & 3.91 & 3.77 & 3.65 & 3.62 & 3.38 & 3.67 \\
\hline $\mathrm{T}_{3}$ Rice starch $(3 \%)$ & 3.95 & 3.82 & 3.71 & 3.65 & 3.41 & 3.71 \\
\hline $\mathrm{T}_{4}$ Cassava starch $(1 \%)$ & 3.81 & 3.65 & 3.57 & 3.50 & 3.30 & 3.56 \\
\hline $\mathrm{T}_{5}$ Cassava starch $(2 \%)$ & 3.86 & 3.71 & 3.58 & 3.53 & 3.32 & 3.60 \\
\hline $\mathrm{T}_{6}$ Cassava starch $(3 \%)$ & 3.89 & 3.75 & 3.62 & 3.59 & 3.34 & 3.64 \\
\hline $\mathrm{T}_{7}$ Neem leaf extract $(10 \%)$ & 3.94 & 3.81 & 3.70 & 3.66 & 3.41 & 3.70 \\
\hline $\mathrm{T}_{8}$ Neem leaf extract $(20 \%)$ & 3.97 & 3.82 & 3.72 & 3.67 & 3.42 & 3.72 \\
\hline $\mathrm{T}_{9}$ Neem leaf extract $(30 \%)$ & 3.99 & 3.86 & 3.73 & 3.68 & 3.43 & 3.74 \\
\hline $\mathrm{T}_{10}$ Control & 3.71 & 3.51 & 3.37 & 3.23 & 2.95 & 3.35 \\
\hline Mean & 3.89 & 3.74 & 3.62 & 3.57 & 3.33 & 3.63 \\
\hline *Initial value (Ascorbic acid) & 4.00 & & & & & \\
\hline \multicolumn{7}{|l|}{$\mathrm{CD}_{0.05}$} \\
\hline $\mathrm{P}$ & & & & & & 0.01 \\
\hline TxP & & & & & & 0.01 \\
\hline PxI & & & & & & 0.01 \\
\hline TxPxI & & & & & & 0.04 \\
\hline
\end{tabular}


Table.6 Effect of various post-harvest treatments on pectin content (as \% calcium pectate) of peach fruit cv. July Elberta fruit during storage at $3 \pm 1^{\circ} \mathrm{C}$

\begin{tabular}{|c|c|c|c|c|c|c|}
\hline \multirow[t]{2}{*}{ Treatments $(\mathrm{T})$} & \multicolumn{6}{|c|}{ Storage interval in days } \\
\hline & 7 & 14 & 21 & 28 & 35 & Mean \\
\hline \multicolumn{7}{|l|}{ Without Pre-cooling $\left(\mathrm{P}_{1}\right)$} \\
\hline $\mathrm{T}_{1}$ Rice starch (1\%) & 2.76 & 2.64 & 2.54 & 2.48 & 2.40 & 2.56 \\
\hline $\mathrm{T}_{2}$ Rice starch $(2 \%)$ & 2.80 & 2.67 & 2.61 & 2.55 & 2.44 & 2.61 \\
\hline $\mathrm{T}_{3}$ Rice starch (3\%) & 2.88 & 2.77 & 2.68 & 2.59 & 2.54 & 2.69 \\
\hline $\mathrm{T}_{4}$ Cassava starch $(1 \%)$ & 2.78 & 2.70 & 2.64 & 2.53 & 2.42 & 2.61 \\
\hline $\mathrm{T}_{5}$ Cassava starch $(2 \%)$ & 2.83 & 2.71 & 2.64 & 3.08 & 2.49 & 2.75 \\
\hline $\mathrm{T}_{6}$ Cassava starch $(3 \%)$ & 2.86 & 2.78 & 2.67 & 2.57 & 2.55 & 2.69 \\
\hline $\mathrm{T}_{7}$ Neem leaf extract $(10 \%)$ & 2.83 & 2.70 & 2.68 & 2.63 & 2.59 & 2.69 \\
\hline $\mathrm{T}_{8}$ Neem leaf extract $(20 \%)$ & 2.86 & 2.73 & 2.71 & 2.65 & 2.59 & 2.71 \\
\hline $\mathrm{T}_{9}$ Neem leaf extract $(30 \%)$ & 2.92 & 2.79 & 2.73 & 2.67 & 2.61 & 2.74 \\
\hline $\mathrm{T}_{10}$ Control & 2.68 & 1.99 & 1.70 & 1.58 & 1.39 & 1.87 \\
\hline Mean & 2.82 & 2.65 & 2.56 & 2.53 & 2.40 & 2.59 \\
\hline \multicolumn{7}{|l|}{ With Pre-cooling $\left(\mathrm{P}_{2}\right)$} \\
\hline $\mathrm{T}_{1}$ Rice starch (1\%) & 2.87 & 2.79 & 2.72 & 2.63 & 2.52 & 2.70 \\
\hline $\mathrm{T}_{2}$ Rice starch $(2 \%)$ & 2.91 & 2.84 & 2.80 & 2.72 & 2.61 & 2.77 \\
\hline $\mathrm{T}_{3}$ Rice starch $(3 \%)$ & 3.01 & 2.95 & 2.85 & 2.79 & 2.71 & 2.86 \\
\hline $\mathrm{T}_{4}$ Cassava starch $(1 \%)$ & 2.87 & 2.82 & 2.71 & 2.62 & 2.52 & 2.71 \\
\hline $\mathrm{T}_{5}$ Cassava starch $(2 \%)$ & 2.90 & 2.87 & 2.76 & 2.72 & 2.66 & 2.78 \\
\hline $\mathrm{T}_{6}$ Cassava starch $(3 \%)$ & 2.94 & 2.90 & 2.83 & 2.78 & 2.71 & 2.83 \\
\hline $\mathrm{T}_{7}$ Neem leaf extract $(10 \%)$ & 2.96 & 2.94 & 2.87 & 2.75 & 2.69 & 2.84 \\
\hline $\mathrm{T}_{8}$ Neem leaf extract $(20 \%)$ & 3.01 & 2.97 & 2.93 & 2.80 & 2.72 & 2.88 \\
\hline $\mathrm{T}_{9}$ Neem leaf extract $(30 \%)$ & 3.03 & 3.01 & 2.96 & 2.80 & 2.78 & 2.92 \\
\hline $\mathrm{T}_{10}$ Control & 2.81 & 2.71 & 2.53 & 2.20 & 2.08 & 2.46 \\
\hline Mean & 2.93 & 2.88 & 2.79 & 2.68 & 2.60 & 2.78 \\
\hline \multicolumn{7}{|c|}{ *Initial value (as \% calcium pectate) 3.06} \\
\hline \multicolumn{7}{|l|}{$\mathrm{CD}_{0.05}$} \\
\hline$P$ & & & & & & 0.03 \\
\hline TxP & & & & & & 0.10 \\
\hline PxI & & & & & & 0.08 \\
\hline TxPxI & & & & & & 0.24 \\
\hline
\end{tabular}

\section{Titratable Acidity (TA)}

A gradual decline in titratable acidity contents was observed with the advancement of storage period under all treatments in both the storage conditions (Table 4). These changes were slower in precooled and coated fruits, especially when 20 and 30 per cent Neem leaf extract 
treatment were used, which could be due to the presence of an active principle compound (azadirachtin) in the extract as it has been reported to be capable of maintaining cellular integrity (Kleeberg, 1996). The postharvest coating with Neem leaf extract $(20 \%)$ which were combined with precooling retained the maximum mean TA content $(0.61 \%)$ that was followed by $30 \%$ Neem leaf extract $\left(\mathrm{T}_{9}\right)$ with $0.60 \%, 2 \%$ Rice starch $\left(\mathrm{T}_{2}\right)$ with $0.60 \%$ and $3 \%$ Rice starch $\left(\mathrm{T}_{3}\right)$ with $0.60 \%$, titratable acidity, respectively; and, further pinpointed that the fruits which were not precooled retained maximum mean TA $(0.60 \%)$ in treatments $3 \%$ Rice starch $\left(\mathrm{T}_{3}\right), 20 \%$ Neem leaf extract $\left(\mathrm{T}_{8}\right)$ with $0.60 \%$ and $30 \%$ Neem leaf extract $\left(\mathrm{T}_{9}\right)$ with $0.60 \%$, respectively; and that the control treatments resulted in retention of the lowest amount of titratable acidity content in fruits. At the same time, the faster rate of decline in acidity in control fruits could be due to the faster metabolic reactions occurring in such fruits as they were neither precooled nor covered with any protective coating material which could have slowed down the physiological changes occurring within them. Nevertheless, the application of coating treatments with rice and Cassava starch, and leaf extracts may also slow down the metabolism of fruits as fruits coated with the materials so used have been reported to maintain higher $\mathrm{CO}_{2}$ and lower $\mathrm{O}_{2}$ levels inside the fruits (Singh and Mohammed, 1997; Kader et al., 1989; Singh et al., 2000 and BenYehoshua, 1985). Similar reported decreases in titratable acidity contents during storage of peach (Vanoli et al., 1995) and apricot (Bayindirli et al., 1995) have confirmed our findings.

\section{Ascorbic acid}

During the present investigations ascorbic acid content of fruits exhibited a decline with an increased in storage intervals (Table 5). The maximum ascorbic acid (3.74 $\mathrm{mg} / 100 \mathrm{~g})$ was retained with a combination of precooling and coating with 30 per cent Neem leaf extract $\left(\mathrm{T}_{9}\right)$ followed by the treatments $20 \%$ Neem leaf extract $\left(\mathrm{T}_{8}\right)$, with $3.72 \mathrm{mg} / 100 \mathrm{~g}, 3 \%$ Rice starch $\left(\mathrm{T}_{3}\right)$ with $3.71 \mathrm{mg} / 100 \mathrm{~g}$ and $10 \%$ Neem leaf extract $\left(\mathrm{T}_{7}\right)$, with $3.70 \mathrm{mg} / 100 \mathrm{~g}$. Das and Dush (1967) reported that decrease in ascorbic acid content in fruits during storage may be due to its degradation during metabolic processes or through enzymatic oxidation of L-ascorbic acid to dehydroascorbic acid. The minimum ascorbic acid content $(2.83 \mathrm{mg} / 100 \mathrm{~g})$ was recorded in the control $\left(\mathrm{T}_{10}\right)$ fruits. High $\mathrm{CO}_{2}$ and low $\mathrm{O}_{2}$ environment that develop inside coated fruits, have also been reported to cause lower activity of peroxidase enzyme which plays an important role in enzymatic degradation of ascorbic acid (Barth et al., 1993). These findings are further supported by the observations of Ulrich (1974), Singh et al., (2000), Singh and Mohammed (1997) and Chauhan (2005) who have also observed similar changes in ascorbic acid levels as a result of coating treatments with Rice starch, Neem leaf extract and Semper fresh coating.

\section{Pectin content}

In general, the pectin content in fruit exhibited a continuous decline with an increased in storage duration under all coating treatments (Table 6). Among all the treatments, the most effective treatment in retaining higher pectin content was found with coating 30 per cent Neem leaf extract $\left(\mathrm{T}_{9}\right)$ as it retained maximum mean pectin content (2.92\%); and it was closely followed by $20 \%$ Neem leaf extract $\left(\mathrm{T}_{8}\right)$ with $2.88 \%$ and $3 \%$ Rice starch $\left(\mathrm{T}_{3}\right)$ with $2.86 \%$. The subsequent loss in pectin content may be due to breakdown of pectin during storage as claimed by Sandhu et al., (1990).

However, such changes were observed to be slower in coated fruit which were also precooled. Such fruits can be expected to retain higher levels of pectin and the degradation of pectin is controlled by the activity of pectic enzymes; the activity of which may be reduced considerably by precooling temperature and coating treatments. The lowest pectin content $(1.87 \%$ as calcium pectate) was recorded in non-precooled control $\left(\mathrm{T}_{10}\right)$ fruits. The gradual 
decrease in pectin content with the advancement of storage period might be the result of pectic enzyme activity on natural pectin in the fruit. These findings are also supported by the observation of Pollard (1975) and Nara et al., (2001) who have also observed similar changes in apple fruits.

Among all the post-harvest edible coating treatments so adopted treatments, 30 per cent neem leaf extract was most effective in minimizing physiological weight loss, firmness, acidity, pectin; and thereby, exhibiting maximum overall acceptability.

\section{Acknowledgements}

The present studies entitled "Studies on shelflife extension of peach [Prunus persica (L.) Batsch] cv. July Elberta" were conducted in the Department of Post-Harvest Technology College of Horticulture, Dr Yashwant Singh Parmar University of Horticulture and Forestry, Nauni, Solan - 173230 (H.P.) and the financial assistance provided by the institution for the study is fully acknowledged.

\section{References}

Anonymous 2014. Statistical Outline of Himachal Pradesh. Department of Economics and Statistics, Shimla, Himachal Pradesh.

AOAC 2000. Official methods of Analysis of the Association of Official Analytical Chemists, Hortwitz, W. (ed.), $16^{\text {th }}$ ed. Association of Official Analytical Chemists, Washington, D.C. USA.

Barth, M.M., Kerbel, E.L., Broussard, S., and Schmidt, S.J.1993. Modified Atmosphere Packaging (High $\mathrm{CO}_{2} /$ Low $\mathrm{O}_{2}$ ) effect on market quality and microbial growth in broccoli spears under temperature abuse condition. Acta Horticultura 343: 187 189.

Bayindrili, L., Sumnu, G., and Kamadan, K. 1995. Effect of semper fresh and Jon fresh fruit coatings on post harvest quality of Satsuma mandarins. Journal of Food Processing and Preservation 19(5): 399407.

Ben-Yehoshua, S. 1985. Individual seal packaging of fruit and vegetable in plastic films- a new post-harvest technique. Horticultural Science 20: 32-37.

Bhardwaj, R. L., and Sen, N.Z. 2003. Zero energy cool chamber storage of Mandrain (Citrus reticulata cv. Nagpur Santra). Journal of Food Science and Technology 40(6): 669-672.

Chauhan, S.K. 2005. Storage behaviour of apple cv. Starking Delicious as affected by pre and postharvest treatments of neem based formulations, plant extract and leaves. Ph.D Thesis, Dr Y S Parmar University of Horticulture and Forestry, Nauni, Solan, $\mathrm{H}$ P, India.

Combrink, J.C., and Visagie, I.R. 1997. Effect of partial cooling prior to packing on the quality of apricots, nectarines, peaches and plums after storage. Deciduous Fruit Grower 47(9): 356-359.

Das, R.C., and Dush, J.1967. The effect of wax emulsion, 2,4-D and 2,4,5-T on storage behavior of Mosambi fruits. Proceeding International Symposium of Subtropical and Tropical Horticulture New Delhi. pp. 104-107.

Gross, K.C., and Sams, C.E. 1984. Changes in the cell wall neutral sugar composition during fruit ripening. A special survey. Phytochemistry 23: 2457-2461.

Kader, A.A., Zagory, D. and Kerbel, E. L. 1989. Modified atmosphere packaging of fruits and vegetables. CRC Crit. Rev. Food Science and Nutrition 88:1-30.

Kleeberg, H.1996. The Neem Azal conception: Future possibilities of the use of neem in biological and integrated pest management. In: Neem and Environment (Eds.) R.P. Singh, M.S. Chavi, R.K. Raheja. Oxford and IBH publication Company Pvt. Ltd., New Delhi. Pp 875882.

Lowings, P.H., and Cutts, D.F. 1982. The preservation of fresh fruits and vegetables. Proceedings of Institute Food 
Science Technology Annual Symposium July 1981, Nottingham, UK.

Nara, K., Kato, Y., and Motomura, Y. 2001. Involvement of terminal arabinose and galactose pectic compounds in mealiness of apple fruit during storage. Postharvest Biological Technology 22(2): 141-150.

NHB 2014. Statistical Database of the National Horticulture Board, Gurgaon. http;//nhb.gov.in/area_production.html

Pollard, I.E. 1975. Pectinolytic enzyme activity and changes in water potential components associated with internal breakdown in McIntosh apple. Journal of American Society for Horticultural Science 100: 647-649.

Ranganna, S.1986. Handbook of Analysis and Quality Control of Fruit and Vegetable Products. $2^{\text {nd }}$ edn. Tata McGraw Hill Publication Company, New Delhi.

Saftner, R.A. 2000. The potential of fruit coating and film treatments for improving the storage and shelf life qualities of 'Gala' and 'Golden Delicious' apple. Journal of American Society for Horticultural Science 124(6): 682-689.

Sandhu, K.S., Bhatia, B.S., and Shukla, F.C.1990. Effect of lye treatment on the quality of kinnow juice. Indian Journal of Horticulture 47:55-59.
Singh, J.N., Acharya, P., and Singh, B.B. 2000. Effect of $\mathrm{GA}_{3}$ and plant extracts on storage behaviour of mango (Mangifera indica) cv. Langra. Haryana Journal of Horticultural Science 29 (3 and 4).

Singh, U.B., and Mohammed, S. 1997. Comparative efficacy of wax emulsion and rice starch on post harvest shelf life of fully ripe guava fruits. Journal of Food Science and Technology 34(6): 519-522.

Smith, S.M., and Stow, J.R. 1984. Potential of a sucrose ester coating material for improving the storage and shelf life qualities of Cox Orange Pippin. Annals of Applied Ecology 104: 383-387

Soodan, A.S., Wafri, B.S., and Kaul, A.K.1994. Peach diversity in Kashmir. Indian Horticulture 32(2): 21-44.

Sornsrivichai, J., Uthaibuthra, J., and Thongaram, A. 1990. Effect of wax coating on storage life and fruit quality of five Asian pear cultivars. Acta Horticultulturae 279: 511-528

Ulrich, R. 1974. Organic acids. In: Biochemistry of fruits and their products (Ed.) A. C. Hulme. Academic Press, New York. Pp 89-118.

Vanoli, M., Visai, C., and Rizzalo, A.1995. Peach quality: influence of ripening and cold storage. Acta Horticulturae 379: 445448.

\section{How to cite this article:}

Sophia Devi, L. and Gulleria, S.P.S. 2018. Effect of Different Post-Harvest Treatments on the Storage Quality of Peach cv. July Elberta. Int.J.Curr.Microbiol.App.Sci. 7(06): 854-865. doi: https://doi.org/10.20546/ijcmas.2018.706.100 